\title{
RELATIONSHIPS BETWEEN MONOTONICITY AND COMPLEX ROTUNDITY PROPERTIES WITH SOME CONSEQUENCES
}

\author{
HENRYK HUDZIK and AGATA NARLOCH
}

\begin{abstract}
It is proved that a point $f$ of the complexification $E^{\mathrm{C}}$ of a real Köthe space $E$ is a complex extreme point if and only if $|f|$ is a point of upper monotonicity in $E$. As a corollary it follows that $E$ is strictly monotone if and only if $E^{\mathrm{C}}$ is complex rotund. It is also shown that $E$ is uniformly monotone if and only if $E^{\mathrm{C}}$ is uniformly complex rotund. Next, the fact that $|x| \in S\left(E^{+}\right)$is a ULUM-point of $E$ whenever $x$ is a C-LUR-point of $S\left(E^{\mathrm{C}}\right)$ is proved, whence the relation that $E$ is a ULUM-space whenever $E^{\mathrm{C}}$ is C-LUR is concluded. In the second part of this paper these general results are applied to characterize complex rotundity of properties Calderón-Lozanovskiı spaces, generalized Calderón-Lozanovskiĭ spaces and Orlicz-Lorentz spaces.
\end{abstract}

\section{Introduction}

First we introduce the notations and define the notions used in this paper. Let $(T, \Sigma, \mu)$ be a complete and $\sigma$-finite measure space and $L^{0}=L^{0}(T, \Sigma, \mu)$ be the space of all (equivalence classes of) $\Sigma$-measurable real functions defined on $T$. A Banach space $\left(E,\|\|_{E}\right)$ is said to be a real Köthe space if $E \subset L^{0}$ and:

(i) for every $x \in L^{0}$ and $y \in E$ with $|x(t)| \leqslant|y(t)| \mu$-a.e. in $T$, we have $x \in E$ and $\|x\|_{E} \leqslant\|y\|_{E}$,

(ii) there is a function $x \in E$ such that $x(t)>0$ for any $t \in T$.

In the whole paper, if a real Köthe space $E$ is fixed, $S(E)$ and $B(E)$ denote the unit sphere and the unit ball of $E$, respectively. The same notations are used for complex Köthe spaces defined bellow. In this paper global and local monotonicity properties are considered for real Köthe spaces only although these properties can be also defined for abstract Banach lattices (see [2]).

We say that $E$ is strictly monotone ( $E \in(S M)$ for short) if for every $x, y$ in the positive cone $E^{+}=\{x \in E: x \geqslant 0\}$ we have $\|x\|_{E}<\|y\|_{E}$ whenever $x \leqslant y$ and $x \neq y$. E is said to be uniformly monotone $(E \in(U M))$

Received October 20, 2003; in revised form January 13, 2004. 
if for every $\varepsilon \in(0,1)$ there is $\delta(\varepsilon) \in(0,1)$ such that $\|x-y\|_{E} \leqslant 1-\delta(\varepsilon)$ whenever $0 \leqslant y \leqslant x,\|x\|_{E}=1$ and $\|y\|_{E} \geqslant \varepsilon$. A point $x \in E^{+}$is called a point of upper monotonicity (UM-point) if for every $y \in E^{+} \backslash\{0\}$ we have $\|x\|_{E}<\|x+y\|_{E}$. We say that $x \in S\left(E^{+}\right):=E^{+} \cap S(E)$ is a point of upper local uniform monotonicity (ULUM-point) if for any sequence $\left(x_{n}\right)$ such that $x \leqslant x_{n}(n \in \mathrm{N})$ there holds $\left\|x-x_{n}\right\|_{E} \rightarrow 0$ if $\left\|x_{n}\right\|_{E} \rightarrow 1$. If every point in $S\left(E^{+}\right)$is a ULUM-point, then we say that $E$ is an upper locally uniformly monotone space (ULUM-space).

We refer to [2] for the definitions of SM and UM, to [16] for various characterizations of the monotonicity properties in Köthe spaces, to [3], [4], [5], [9], [10], [11], [12], [14], [15], [16], [17], [19] and [20] for criteria of monotonicity properties in various classes of Köthe spaces and to [1] for the application of the uniform monotonicity in ergodic theory.

We say that the Köthe space $E$ has Fatou property $(E \in(F P)$ for short) if for any $x \in L^{0}$ and $\left(x_{n}\right)$ in $E^{+}$such that $x_{n} \uparrow x \mu$-a.e. and $\sup _{n}\left\|x_{n}\right\|_{E}<\infty$, we have $x \in E$ and $\left\|x_{n}\right\|_{E} \rightarrow\|x\|_{E}$.

The Köthe space $E$ is said to be order continuous $(E \in(O C)$ for short) if for any $x \in E$ and any sequence $\left(x_{n}\right)$ in $E^{+}$such that $x_{n} \leqslant|x|(n \in \mathrm{N})$ and $x_{n} \downarrow 0 \mu$-a.e., we have $\left\|x_{n}\right\|_{E} \rightarrow 0$.

For a real Köthe space $\left(E,\|\|_{E}\right)$ we define its complexification

$$
E^{\mathrm{C}}=\{f: T \rightarrow \mathrm{C}: f=x+i y \text { with } x, y \in E\}
$$

endowed with the norm

$$
\|f\|=\left\|\sqrt{x^{2}+y^{2}}\right\|_{E}=\||f|\|_{E} .
$$

The space $\left(E^{\mathrm{C}},\|\|\right)$ is called in this paper a complex Köthe space. If $(F,\|\|)$ is a complex Köthe space, then the space

$$
F_{r}=\{f \in F: \operatorname{Im}(f)=0\}
$$

under the norm induced from $F$ is a real Köthe space. It is easy to see that $E=\left(E^{\mathcal{C}}\right)_{r}$ and $F=\left(F_{r}\right)^{\complement}$ for any real Köthe space $E$ and any complex Köthe space $F$.

Let $X$ be a complex Banach space and $S(X)$ be the unit sphere in $X$. A point $x \in S(X)$ is called a complex extreme point (C-extreme point or $x \in \mathrm{C}$-Ext $(X)$ for short) if for any $y \in X$ with $y \neq 0$ there holds $\sup _{|\lambda| \leqslant 1}\|x+\lambda y\|_{X}>1$. A complex Banach space $X$ is said to be complex rotund (C-rotund) if every $x \in S(X)$ is a C-extreme point. Considering complex local or global rotundity properties of a Banach space $X$, we always assume that $X$ is a complex Banach space. We say that $X$ is uniformly complex rotund (uniformly C-rotund) if for 
every $\varepsilon \in(0,1)$ there exists $\delta(\varepsilon) \in(0,1)$ such that $\|x\|_{X} \leqslant 1-\delta(\varepsilon)$ whenever $\|y\|_{X} \geqslant \varepsilon$ and $\sup _{|\lambda| \leqslant 1}\|x+\lambda y\|_{X} \leqslant 1$. This definition is equivalent to the following one:

$$
\forall_{\varepsilon>0} \exists_{\delta(\varepsilon)>0} \forall_{x, y \in X} \quad\|x\|=1,\|y\| \geqslant \varepsilon \Rightarrow \sup _{|\lambda| \leqslant 1}\|x+\lambda y\| \geqslant 1+\delta(\varepsilon) .
$$

A point $x \in S(X)$ is called a point of complex local uniform rotundity (C-LURpoint $)$ if for every $\varepsilon>0$ there exists $\delta(x, \varepsilon)>0$ such that $\sup _{|\lambda| \leqslant 1}\|x+\lambda y\| \geqslant$ $1+\delta(x, \varepsilon)$ for every $y \in X$ satisfying $\|y\| \geqslant \varepsilon$. If every point of the unit sphere of $X$ is a C-LUR-point, then $X$ is called a C-LUR-space.

The notion of complex rotundity was introduced by Thorp and Whitley in [23], where they showed that the complex space $L^{1}(\Sigma, \mu)$ is C-rotund. Globevnik [13] introduced the notion of uniform C-rotundity and showed that the complex space $L^{1}(\Sigma, \mu)$ has this property. Next Wang and Teng [27] introduced the notion of locally uniform C-rotundity, obtaining criteria for this property in the class of Musielak-Orlicz spaces.

It is well known that monotonicity properties have various applications, among others in ergodic theory (see [1]) and in dominated best approximation problems (see [17] and [19]). Complex rotundity properties have applications in the theory of vector-valued analytic functions. It is known that if $f$ is a function from the unit disc $B(C)$ (in the field of complex numbers $C$ ) into a complex Banach space $X$ and $f$ is analytic, i.e. $x^{*} \circ f$ is analytic in the classical sense for any $x^{*} \in X^{*}$ (the dual space of $X$ ) and the maximum of the function $F(z)=\|f(z)\|$ is attained in an interior point $z_{0} \in B(\mathrm{C})$, then $F$ is a constant function. But, in the case when $X$ is C-rotund, more can be deduced, namely that $f$ is a constant function (see [23]).

From our results it follows that for any real Köthe space $E$ strict monotonicity and uniform monotonicity of $E$ coincide, respectively, with complex rotundity and complex uniform rotundity of $E^{\mathrm{C}}$. On the basis of this observation, most of the results from [24], [25] and [26] concerning complex rotundity properties can be easily deduced from the results of [17], [19] and [20]. In section 2 some other results are also deduced.

\section{Results}

THEOREM 1. For any real Köthe space $E$ a point $f \in S\left(E^{\mathrm{C}}\right)$ is a C-extreme point of $B\left(E^{\mathrm{C}}\right)$ if and only if $|f|$ is a UM-point in $E$.

Proof. For the sufficiency let us pick $f \in S\left(E^{\mathrm{C}}\right), g \in E^{\mathrm{C}}$ and suppose that $|f|$ is a UM-point and $\|f+\lambda g\| \leqslant 1$ for all $\lambda \in \mathrm{C}$ with $|\lambda| \leqslant 1$. We need to show that $g=0$. First we show that $g(t)=0$ for $\mu$-a.e. $t \in T \backslash G$, where 
$G=\operatorname{supp} f$. We have the inequality

$$
\begin{aligned}
& 1=\|f\|=\||f|\|_{E} \leqslant\left\||f|+|g| \chi_{T \backslash G}\right\|_{E} \\
& \leqslant\left\|\frac{1}{2}\left|f+g \chi_{G}\right|+\frac{1}{2}\left|f-g \chi_{G}\right|+|g| \chi_{T \backslash G}\right\|_{E}=\frac{1}{2}\||f+g|+|f-g|\|_{E} \\
& \quad \leqslant \frac{1}{2}\||f+g|\|_{E}+\frac{1}{2}\||f-g|\|_{E}=\frac{1}{2}\|f+g\|+\frac{1}{2}\|f-g\| \leqslant 1 .
\end{aligned}
$$

Then, by the upper monotonicity of $|f|$, we have $g \chi_{T \backslash G}=0$. This means that $\operatorname{supp} g \subset \operatorname{supp} f$. Therefore, to finish the proof of the sufficiency, we need only to show that the set

$$
S=\{t \in T: f(t) \neq 0 \text { and } g(t) \neq 0\}
$$

is a nullset. For every $|\lambda| \leqslant 1$, we have

$$
\begin{aligned}
2=\|2 f\|= & \||f+\lambda g+f-\lambda g|\|_{E} \\
& \leqslant\||f+\lambda g|+|f-\lambda g|\|_{E} \leqslant\|f+\lambda g\|+\|f-\lambda g\| \leqslant 2 .
\end{aligned}
$$

Hence the assumption that $|f|$ is a UM-point yields that

$$
|(f(t)+\lambda g(t))+(f(t)-\lambda g(t))|=|f(t)+\lambda g(t)|+|f(t)-\lambda g(t)|
$$

for $\mu$-a.e. $t \in T$ and, in consequence, $\arg (f(t)+\lambda g(t))=\arg (f(t)-\lambda g(t))+$ $2 k \pi, k \in \mathbf{Z}$. Therefore, the function $h_{\lambda}: \operatorname{supp}(f+\lambda g) \cap \operatorname{supp}(f-\lambda g) \rightarrow \mathbf{R}$, where

$$
h_{\lambda}(t)=\frac{f(t)+\lambda g(t)}{f(t)-\lambda g(t)}
$$

has positive values for every $\lambda \in \mathrm{C}$ with $|\lambda| \leqslant 1$. Let us define $A_{1}=\{t \in$ $S: f(t)+g(t) \neq 0\}, A_{2}=\{t \in S: f(t)-g(t) \neq 0\}, A_{3}=\{t \in S:$ $f(t)+i g(t) \neq 0\}, A_{4}=\{t \in S: f(t)-i g(t) \neq 0\}$ and $B_{j}=S \backslash A_{j}$ for $j \in\{1,2,3,4\}$. We see at once that $B_{k} \cap B_{l}=\emptyset$ for $k \neq l$ and $k, l \in\{1,2,3,4\}$.

Let us take now arbitrary $t \in B_{1}$. Since $B_{1} \subset A_{3} \cap A_{4}$, so

$$
i g(t)=\frac{f(t)\left(h_{i}(t)-1\right)}{h_{i}(t)+1} \quad \text { and } \quad f(t)+g(t)=0
$$

for any $t \in B_{1}$. This implies that

$$
i=\frac{1-h_{i}(t)}{h_{i}(t)+1} \in \mathrm{R}
$$

for any $t \in B_{1}$, which is a contradiction. So $B_{1}=\emptyset$. We can prove in a similar way that $B_{2}=B_{3}=B_{4}=\emptyset$. 
Next for any $t \in A:=\bigcap_{k=1}^{4} A_{k}$, by (1), we have

$$
g(t)=\frac{f(t)\left(h_{1}(t)-1\right)}{h_{1}(t)+1},
$$

because $t \in A_{1} \cap A_{2}$. So, we see that

$$
g(t) \bar{f}(t)=\frac{|f(t)|^{2}\left(h_{1}(t)-1\right)}{h_{1}(t)+1} \in \mathbf{R} .
$$

But since we also have $t \in A_{3} \cap A_{4}$ for $t \in A$, we get

$$
i g(t)=\frac{f(t)\left(h_{i}(t)-1\right)}{h_{i}(t)+1},
$$

and so the condition

$$
i g(t) \bar{f}(t)=\frac{|f(t)|^{2}\left(h_{i}(t)-1\right)}{h_{i}(t)+1} \in \mathbf{R}
$$

follows. Properties (2) and (3) show that $g(t) \bar{f}(t)=0$ for any $t \in \bigcap_{k=1}^{4} A_{k}$. Since $\bar{f}(t) \neq 0$ for $t \in \bigcap_{k=1}^{4} A_{k}$, we get $g(t)=0$ for such $t$, a contradiction. Therefore $\bigcap_{k=1}^{4} A_{k}=\emptyset$. Consequently, $S=\bigcap_{k=1}^{4} A_{k} \cup \bigcup_{k=1}^{4} B_{k}=\emptyset$.

Necessity. Suppose that $|f|$ is not a UM-point. Then there exist $y \in E^{+}$ such that $|f| \leqslant y,|f| \neq y$ and $\||f|\|_{E}=\|y\|_{E}=1$. By Lemma 1 from [6], $|f|$ cannot be an extreme point of the unit ball in $E$, so there is $g \in E^{+} \backslash\{0\}$ such that $\||f| \pm g\|_{E}=1$. Consequently,

$$
\|f+\lambda g\|=\||f+\lambda g|\|_{E} \leqslant\||f|+g\|_{E}=1
$$

for any $|\lambda| \leqslant 1$. Hence $f \notin \mathrm{C}$-Ext $\left(E^{\mathrm{C}}\right)$.

A real Köthe space $E$ is strictly monotone if and only if every point in $S\left(E^{+}\right)$is a UM-point. Therefore we have

Corollary 1. For any real Köthe space E, its complexification $E^{\mathrm{C}}$ is a $C$-rotund space if and only if $E$ is strictly monotone.

THEOREM 2. For any real Köthe space $E$, the space $E^{\mathrm{C}}$ is uniformly $\mathrm{C}$-rotund if and only if $E$ is uniformly monotone.

Proof. Necessity. Suppose that $E$ is not a uniformly monotone space. Theorem 6 in [16] shows that there exist sequences $\left(x_{n}\right),\left(y_{n}\right) \subset E^{+}$and a number $\varepsilon>0$ such that

(i) $\left\|x_{n}\right\|_{E}=1$, 
(ii) $\left\|y_{n}\right\|_{E} \geqslant \varepsilon$,

(iii) $\left\|x_{n}+y_{n}\right\|_{E} \leqslant 1+\frac{1}{n}$

for every $n \in \mathrm{N}$. We have $\left\|x_{n}\right\|=1=\left\|x_{n}\right\|_{E}$ for any $n \in \mathrm{N}$ and

$$
\left\|x_{n}+\lambda y_{n}\right\| \leqslant\left\|\left|x_{n}\right|+\left|y_{n}\right|\right\|_{E}=\left\|x_{n}+y_{n}\right\|_{E} \leqslant 1+\frac{1}{n}
$$

for any $\lambda \in \mathrm{C}$ satisfying $|\lambda| \leqslant 1$. Hence $\sup _{|\lambda| \leqslant 1}\left\|x_{n}+\lambda y_{n}\right\| \leqslant 1+\frac{1}{n}$ for any $n \in N$, which means that $E^{\mathrm{C}}$ is not uniformly C-rotund.

Sufficiency. Let us assume that $E$ is uniformly monotone. Let $f, g \in E^{\mathrm{C}}$, $\varepsilon$ be arbitrary positive number less than 1 and $\|g\| \geqslant \varepsilon$. Suppose that $\sup _{|\lambda| \leqslant 1}$ $\|f+\lambda g\| \leqslant 1$. We will show that there exists a number $\delta \in(0,1)$, depending only on $\varepsilon$, such that $\|f\| \leqslant 1-\delta$. Put $\mathscr{K}=\{1,-1, i,-i\}$. Proposition 5.17 in [5] implies that there exists a number $\beta \in(0,1)$ (depending only on $\varepsilon$ ) such that the following implication is true for arbitrary $u, v \in \mathrm{C}$ :

$$
|u| \geqslant \frac{\varepsilon}{8} \max _{k \in \mathscr{K}}|v+k u| \Longrightarrow|v| \leqslant \frac{1-\beta}{4} \sum_{k \in \mathscr{K}}|v+k u| .
$$

Let $A=\left\{t \in T:|g(t)| \geqslant \frac{\varepsilon}{8} \max _{k \in \mathscr{K}}|f(t)+k g(t)|\right\}$. For every $t \in T \backslash A$ we have

$$
\frac{8}{\varepsilon}|g(t)|<\max _{k \in \mathscr{K}}|f(t)+k g(t)|,
$$

so, taking into account the assumption that $\sup _{|\lambda| \leqslant 1}\|f+\lambda g\| \leqslant 1$, we get

$$
\begin{aligned}
\left\|g \chi_{T \backslash A}\right\|=\frac{\varepsilon}{8}\left\|\frac{8}{\varepsilon}|g| \chi_{T \backslash A}\right\|_{E} \leqslant \frac{\varepsilon}{8} \| \max _{k \in \mathscr{K}} \mid & f\left(k g \mid \chi_{T \backslash A} \|_{E}\right. \\
\leqslant & \frac{\varepsilon}{8} \sum_{k \in \mathscr{K}}\left\||f+k g| \chi_{T \backslash A}\right\|_{E} \leqslant \frac{\varepsilon}{2} .
\end{aligned}
$$

Since $\|g\| \geqslant \varepsilon$, by the triangle inequality, it follows that

$$
\left\|g \chi_{A}\right\| \geqslant \frac{\varepsilon}{2}
$$

By the fact that the set $\mathscr{K}$ is equal to its own inverse, $\left\{\frac{1}{k}: k \in \mathscr{K}\right\}$, it follows that

$$
\begin{aligned}
|g(t)| \leqslant \frac{1}{4} \sum_{k \in \mathscr{H}} \mid g(t) & +k f(t) \mid \\
& =\frac{1}{4} \sum_{k \in \mathscr{K}}|k|\left|\frac{1}{k} g(t)+f(t)\right| \leqslant \frac{1}{4} \sum_{k \in \mathscr{K}}|f(t)+k g(t)|
\end{aligned}
$$


for any $t \in A$, whence

$$
\frac{\varepsilon}{2} \leqslant\left\|g \chi_{A}\right\| \leqslant\left\|\frac{1}{4} \sum_{k \in \mathscr{K}}|f+k g| \chi_{A}\right\|_{E} .
$$

Consequently,

$$
\left\|\frac{\beta}{4} \sum_{k \in \mathscr{K}}|f+k g| \chi_{A}\right\|_{E} \geqslant \frac{\varepsilon \beta}{2} .
$$

By implication (4) and the definition of the set $A$, we have

$$
\begin{aligned}
& \|f\|=\left\||f| \chi_{T \backslash A}+|f| \chi_{A}\right\|_{E} \\
& \leqslant\left\|\frac{1}{4} \sum_{k \in \mathscr{K}}|f+k g| \chi_{T \backslash A}+\frac{1-\beta}{4} \sum_{k \in \mathscr{K}}|f+k g| \chi_{A}\right\|_{E} \\
& \quad=\left\|\frac{1}{4} \sum_{k \in \mathscr{K}}|f+k g| \chi_{T}-\frac{\beta}{4} \sum_{k \in \mathscr{K}}|f+k g| \chi_{A}\right\|_{E} .
\end{aligned}
$$

Now properties (5) and (6) and uniform monotonicity of $E$ show that there exists $\delta>0$, depending only on $\varepsilon$, such that $\|f\| \leqslant 1-\delta$.

LEMMA 1. Let $E$ be any real Köthe space. If $x \in S\left(E^{\complement}\right)$ is a C-LUR-point, then $|x|$ is a ULUM-point in $E$.

Proof. It is obvious that $|x| \in S(E)$ whenever $x \in S\left(E^{\mathrm{C}}\right)$. Suppose that $|x|$ is not a ULUM-point. Then there exists a sequence $\left(y_{n}\right) \subset E^{+}$satisfying $\left\|y_{n}\right\|_{E} \rightarrow 1,|x| \leqslant y_{n}$ and $\left\|y_{n}-|x|\right\|_{E} \geqslant \delta$ for any $n \in \mathbf{N}$ and some $\delta>0$. If we denote $z_{n}=y_{n}-|x|$, then we have $\left\|z_{n}\right\| \geqslant \delta$ and

$$
\begin{aligned}
1 \leqslant \sup _{|\lambda| \leqslant 1}\left\|x+\lambda z_{n}\right\| & \leqslant \sup _{|\lambda| \leqslant 1}\left\||x|+\left|\lambda z_{n}\right|\right\|_{E} \\
& =\left\||x|+\left|z_{n}\right|\right\|_{E}=\left\||x|+y_{n}-|x|\right\|_{E}=\left\|y_{n}\right\|_{E} \rightarrow 1 .
\end{aligned}
$$

This means that $x$ is not a C-LUR-point.

COROLlary 2. If $E$ is a real Köthe space such that $E^{\mathrm{C}}$ is a C-LUR space, then $E$ is a ULUM-space.

\section{Some consequences}

In this section we will apply the results of the first section to get some results on the complex rotundity properties in Orlicz-Lorentz spaces, CalderónLozanovskiı̌ spaces and generalized Calderón-Lozanovskiŭ spaces. To do this 
we will also complete results from [14] on the monotonicity properties of Lorentz spaces and we will extend them to Orlicz-Lorentz spaces.

A function $\varphi: T \times \mathrm{R} \rightarrow[0, \infty]$ is said to be a Musielak-Orlicz function if $\varphi(t,$.$) is convex, even, vanishing and continuous at zero, not identically equal$ to zero for $\mu$-a.e. $t \in T$ and $\varphi(., u)$ is a $\Sigma$-measurable function for any $u \in \mathbf{R}$. If $\sup \{u \geqslant 0: \varphi(t, u)=0\}=0$ for $\mu$-a.e. $t \in T$, then we will write $\varphi>0$. If $\varphi(t,$.$) takes only finite values for \mu$-a.e. $t \in T$, then we will write $\varphi<\infty$. For a Musielak-Orlicz function $\varphi$ and $x \in L^{0}$, we define $\varphi \circ x(t)=\varphi(t, x(t))$.

Given a real Köthe space $E$ and a Musielak-Orlicz function $\varphi$, we define on $L^{0}$ the convex semimodular

$$
\varrho_{\varphi}^{E}(x)= \begin{cases}\|\varphi \circ|x|\|_{E}, & \text { if } \varphi \circ|x| \in E \\ \infty, & \text { otherwise. }\end{cases}
$$

The generalized Calderón-Lozanovskil space $E_{\varphi}$ generated by the couple $(E, \varphi)$ is defined as the set of those $x \in L^{0}$ such that $\varrho_{\varphi}^{E}(\lambda x)<+\infty$ for some $\lambda>0$. The norm in $E_{\varphi}$ is defined by

$$
\|x\|_{\varphi}^{E}=\inf \left\{\lambda>0: \varrho_{\varphi}^{E}(x / \lambda) \leqslant 1\right\} .
$$

If $\varphi$ does not depend on $t \in T$, that is, $\varphi$ is an Orlicz function, then $E_{\varphi}$ is called the Calderón-Lozanovskil space. If $E$ has the Fatou property, then $E_{\varphi}$ also has this property, whence $E_{\varphi}$ is a Banach space (see [11]).

The generalized Calderón-Lozanovskiŭ space can be complex or real according to whether one considers real or complex $L^{0}$. Denote by $E_{\varphi}^{\mathrm{C}}$ and $E_{\varphi}$ the complex and the real Calderón-Lozanovskiı spaces, respectively. It is obvious that $E_{\varphi}^{\mathrm{C}}=\left(E_{\varphi}\right)^{\mathrm{C}}$, that is, $E_{\varphi}^{\mathrm{C}}$ is the complexification of real $E_{\varphi}$.

In this section we will consider C-rotundity and uniform C-rotundity of generalized Calderón-Lozanovskiĭ spaces. We will restrict ourselves to the cases of a non-atomic measure space and counting measure space $\left(\mathrm{N}, 2^{\mathrm{N}}, \mu\right)$, where $\mu(A)=\operatorname{Card}(A)$. It is well known that any $\sigma$-finite measure space is the direct sum of two measure spaces $\left(A, \Sigma \cap A,\left.\mu\right|_{A}\right)$ and $\left(B, \Sigma \cap B,\left.\mu\right|_{B}\right)$, where $A \cap B=\emptyset, A \cup B=T,\left(A, \Sigma \cap A,\left.\mu\right|_{A}\right)$ is a non-atomic measure space, $\left(B, \Sigma \cap B,\left.\mu\right|_{B}\right)$ is a purely atomic measure space with $B$ being a finite or infinite counting set (so, if $B$ is infinite, we can identify $B$ with $\mathrm{N}$ and $\Sigma \cap B$ with $2^{\mathrm{N}}$ ). The generalized Calderón-Lozanovskiu space $E_{\varphi}$ over a $\sigma$-finite measure space $(T, \Sigma, \mu)$ can be written as the direct sum $E_{\varphi}\left(A, \Sigma \cap A,\left.\mu\right|_{A}\right) \oplus E_{\varphi}\left(B, \Sigma \cap B,\left.\mu\right|_{B}\right)$. Consequently, if $E_{\varphi}(T, \Sigma, \mu)$ has an appropriate monotonicity property (analogously $E_{\varphi}^{\mathrm{C}}(T, \Sigma, \mu)$ has an appropriate C-rotundity property) then both $E_{\varphi}\left(A, \Sigma \cap A,\left.\mu\right|_{A}\right)$ and $E_{\varphi}\left(B, \Sigma \cap B,\left.\mu\right|_{B}\right)$ (analogously $E_{\varphi}^{C}\left(A, \Sigma \cap A,\left.\mu\right|_{A}\right)$ and $E_{\varphi}^{C}\left(B, \Sigma \cap B,\left.\mu\right|_{B}\right)$ ) have appropriate properties if they are considered with the norm induced from the whole space. 
Conversely, if criteria for the monotonicity (resp. C-rotundity) properties are known for both parts separately, then it is possible to deduce respective criteria for the whole space although it is not automatic because it can happen that both parts of the space (over $A$ and over $B$ ) have a fixed geometric property but the whole space has not. Moreover, in the case of generalized CalderónLozanovskiĭ spaces, we may assume without loss of generality that the purely atomic part of $T$ is the counting measure space $\left(\mathrm{N}, 2^{\mathrm{N}}, \mu\right)$. Otherwise, instead of a Musielak-Orlicz function $\varphi=\left(\varphi_{i}\right)_{i=1}^{\infty}$ we consider the Musielak-Orlicz function $\psi=\left(\psi_{i}\right)_{i=1}^{\infty}$ with $\psi_{i}(u)=\varphi_{i}(u) \mu(\{i\})$ and the counting measure space. For this reason, generalized Calderón-Lozanovskiu spaces may be considered over a non-atomic measure space and over the counting measure space only.

In the case of a non-atomic measure space we say a Musielak-Orlicz function $\varphi$ satisfies the $\Delta_{2}^{E}$-condition ( $\varphi \in \Delta_{2}^{E}$ for short) if there are a positive constant $K>0$ and a nonnegative function $h \in L^{0}$ such that $\varphi \circ h \in E$ and $\varphi(t, 2 u) \leqslant K \varphi(t, u)$ for $\mu$-a.e. $t \in T$ and all $u \geqslant h(t)$ (see [11]).

Theorem 3. Let $(T, \Sigma, \mu)$ be a non-atomic $\sigma$-finite complete measure space, $E$ be an order continuous real Köthe function space with the Fatou property and $\varphi$ be a Musielak-Orlicz function. Then the generalized CalderónLozanovskiu space $E_{\varphi}^{\mathrm{C}}$ is C-rotund if and only if $\varphi>0, \varphi \in \Delta_{2}^{E}$ and $E$ is strictly monotone space.

Proof. The theorem follows by Corollary 1 and Theorem 1 in [10], where criteria for strict monotonicity of $E_{\varphi}$ are presented.

We say an Orlicz function $\varphi$ satisfies condition $\Delta_{2}(0)\left(\varphi \in \Delta_{2}(0)\right)$ if there exist $K>0, u_{0}>0$ such that $0<\varphi\left(u_{0}\right)$ and the inequality

$$
\varphi(2 u) \leqslant K \varphi(u)
$$

holds for $u \in\left[0, u_{0}\right]$. We say, the function $\varphi$ satisfies condition $\Delta_{2}(\infty)(\varphi \in$ $\left.\Delta_{2}(\infty)\right)$ if there exist $K>0, u_{0}>0$ such that $\varphi\left(u_{0}\right)<\infty$ and inequality (7) holds for all $u \geqslant u_{0}$. If there exists $K>0$ such that $\varphi(2 u) \leqslant K \varphi(u)$ for all $u \geqslant 0$, then we say that $\varphi$ satisfies condition $\Delta_{2}\left(\mathbf{R}_{+}\right)\left(\varphi \in \Delta_{2}\left(\mathbf{R}_{+}\right)\right)$.

Lemma 2. If $(T, \Sigma, \mu)$ is a non-atomic $\sigma$-finite complete measure space, $E$ is a real Köthe space over the measure space $(T, \Sigma, \mu)$ and $\varphi$ is a MusielakOrlicz function not depending on the parameter $t \in T$, that is, $\varphi$ is an Orlicz function, then $\varphi \in \Delta_{2}^{E}$ is equivalent to:

(a) $\varphi \in \Delta_{2}(\infty)$ if $L^{\infty} \hookrightarrow E$,

(b) $\varphi \in \Delta_{2}\left(\mathbf{R}_{+}\right)$if $L^{\infty} \hookrightarrow$. 
Proof. Let us first prove (a). Assume $\varphi \in \Delta_{2}^{E}$, that is, there is a constant $K>0$, a set $A \in \Sigma$ with $\mu(A)=0$ and a nonnegative function $h \in L^{0}$ such that $\varphi \circ h \in E$ and $\varphi(2 u) \leqslant K \varphi(u)$ for all $t \in T \backslash A$ and $u \geqslant h(t)$. Consequently, $\varphi(2 u) \leqslant K \varphi(u)$ for all $u \geqslant \inf _{t \in T \backslash A} h(t)$. It is obvious that $a:=\inf _{t \in T \backslash A} h(t)<\infty$ and $\varphi(a)<\infty$, whence we get that $\varphi \in \Delta_{2}(\infty)$. On the other hand, assuming that $\varphi(2 u) \leqslant K \varphi(u)$ for some $K>0$ and any $u \geqslant u_{0}$, where $u_{0}>0$ is such that $\varphi\left(u_{0}\right)<\infty$, by $L^{\infty} \hookrightarrow E$, we have $\varphi\left(u_{0}\right) \chi_{T} \in E$, so $\varphi \in \Delta_{2}^{E}$. This means that (a) has been proved.

Let us prove (b). Let $\varphi \in \Delta_{2}^{E}$ and $K, h$ be as in the definition of the condition $\Delta_{2}^{E}$. Then for every $t \in T \backslash A$ with $\mu(A)=0$ we have $\varphi(2 u) \leqslant K \varphi(u)$ if $u \geqslant h(t)$ and

$$
\varphi(2 u) \leqslant \varphi(2 h(t)) \leqslant K \varphi(h(t)) \leqslant K \varphi(u)+K \varphi(h(t))
$$

if $u \in[0, h(t)]$. Consequently,

$$
\varphi(2 u) \leqslant K \varphi(u)+K \inf _{t \in T \backslash A} \varphi(h(t))
$$

for every $u \in \mathbf{R}_{+}$. We need to prove that $a:=\inf _{t \in T \backslash A} \varphi(h(t))=0$. Assume on the contrary that $a \neq 0$ and define $g=a \chi_{T \backslash A}$. Then $g \leqslant \varphi \circ h \chi_{T \backslash A}$. Since $L^{\infty} \hookrightarrow E$, we have $g \notin E$ and so $\varphi \circ h \chi_{T \backslash A} \notin E$, a contradiction.

It is obvious that $\varphi \in \Delta_{2}\left(\mathbf{R}_{+}\right)$implies that $\varphi \in \Delta_{2}^{E}$, so (b) is proved.

COROLlary 3. Let $E$ be a real Köthe function space with the Fatou property and with an order continuous norm, and let $\varphi$ be an Orlicz function. Then the Calderón-Lozanovskiu space $E_{\varphi}^{\mathrm{C}}$ is C-rotund if and only if $\varphi>0, \varphi \in \Delta_{2}^{E}$ and $E$ is a strictly monotone space.

Proof. This is a consequence of Theorem 3. Note that by Lemma 2, for $\varphi$ being an Orlicz function $\varphi \in \Delta_{2}^{E}$ has a simpler meaning than in the case when $\varphi$ is a Musielak-Orlicz function. By Lemma 2, we can apply Theorems 1 and 2 from [15] to get $\varphi \in \Delta_{2}^{E}$. Next, $\varphi \in \Delta_{2}^{E}$ implies that $\varphi<\infty$. From Theorem 1 in [4] it follows that if $\varphi<\infty$ and $E_{\varphi} \in(S M)$ (that is, if $E_{\varphi}^{\mathrm{C}}$ is C-rotund), then $\varphi>0$ and $E \in(S M)$.

The sufficiency follows from Theorem 1 in [4] and our Corollary 1.

In the sequence case, that is, in the case of counting measure and a Köthe sequence space $e$, we say that a Musielak-Orlicz function $\varphi=\left(\varphi_{n}\right)_{n=1}^{\infty}$ satisfies condition $\delta_{2}^{e}\left(\varphi \in \delta_{2}^{e}\right.$ for short $)$ if there exist constants $a, K>0$ and a sequence $c=\left(c_{n}\right)_{n=1}^{\infty}$ in $e^{+}$such that for all $n \in \mathrm{N}$ and $u \in \mathbf{R}_{+}$the inequality $\varphi_{n}(2 u) \leqslant$ $K \varphi_{n}(u)+c_{n}$ holds whenever $\left\|\varphi_{n}(u) e_{n}\right\|_{e} \leqslant a$, where we put $e_{n}=\chi_{\{n\}}$ for $n \in \mathrm{N}$. 
We will say that $\varphi=\left(\varphi_{n}\right)_{n=1}^{\infty}$ satisfies condition ( +$)$ if for any $i \in \mathrm{N}$ there is $u_{i}>0$ such that $\left\|\varphi\left(u_{i}\right) e_{i}\right\|_{e}=1$.

THeOREM 4. Let e be a real Köthe sequence space with the Fatou property and $\varphi$ be a Musielak-Orlicz function. The generalized Calderón-Lozanovski space $e_{\varphi}^{\mathrm{C}}$ is C-rotund if and only if $\varphi>0, \varphi \in \delta_{2}^{e}, \varphi$ satisfies the condition (+) and $e$ is strictly monotone.

Proof. This is a consequence of our Corollary 1 and Theorem 5.1 in [12] provided we can prove the necessity of the condition $(+)$. So, let us prove this. Assume on the contrary that the condition $(+)$ is not satisfied, that is, $\left\|\varphi_{j}\left(a_{j}\right) e_{j}\right\|_{e}<1$ for some $j \in \mathrm{N}$, where $a_{j}=\sup \left\{u \geqslant 0: \varphi_{j}(u)<\infty\right\}$. Define $x=a_{j} e_{j}$ and $y=x+b e_{l}$, where $l \neq j$ and $b>0$ is so small that $\left\|\varphi_{j}\left(a_{j}\right) e_{j}\right\|_{e}+\left\|\varphi_{l}(b) e_{l}\right\|_{e} \leqslant 1$. Then we easily get that $\|x\|_{\varphi}^{e}=\|y\|_{\varphi}^{e}=1$. Since $x \neq y$, this means that the space $e_{\varphi}$ is not strictly monotone.

Let us say that $\varphi=\left(\varphi_{n}\right)_{n=1}^{\infty}$ satisfies condition $D_{2}^{e}$ if there are positive constants $a$ and $K$ and sequences $\left(a_{n}\right)_{n=1}^{\infty}$ and $\left(b_{n}\right)_{n=1}^{\infty}$ of positive numbers such that $a_{n}<b_{n}$ and $\varphi_{n}\left(b_{n}\right)\left\|e_{n}\right\|_{e}=a$ for all $n \in \mathrm{N},\left(\varphi_{n}\left(a_{n}\right)\right)_{n=1}^{\infty} \in e$ and $\varphi_{n}(2 u) \leqslant K \varphi_{n}(u)$ for all $n \in \mathrm{N}$ and $u \in\left[a_{n}, b_{n}\right]$.

Remark 1. Note that if for all functions $\varphi_{n}$ defining the function $\varphi$ we have $a_{\varphi_{n}}<b_{\varphi_{n}} / 2$, where $a_{\varphi_{n}}=\sup \left\{u \geqslant 0: \varphi_{n}(u)=0\right\}$ and $b_{\varphi_{n}}=\sup \{u \geqslant 0$ : $\left.\varphi_{n}(u)<\infty\right\}$, then $\varphi=\left(\varphi_{n}\right)_{n=1}^{\infty} \in \delta_{2}^{e}$ iff $\left(\varphi_{n}\right)_{n>k} \in \delta_{2}^{e}$ for some (for any) $k \in \mathrm{N}$ and $\varphi=\left(\varphi_{n}\right)_{n=1}^{\infty} \in D_{2}^{e}$ iff $\varphi=\left(\varphi_{n}\right)_{n>k} \in D_{2}^{e}$ for some (for any) $k \in \mathrm{N}$.

Lemma 3. Let $\varphi=\left(\varphi_{n}\right)_{n=1}^{\infty}$ be a Musielak-Orlicz function and e be a real sequence Köthe space. Then $\varphi \in D_{2}^{e}$ implies $\varphi \in \delta_{2}^{e}$. If in addition e is continuously embedded into $c_{0}\left\{\left\|e_{n}\right\|_{e}\right\}$, then $\varphi \in \delta_{2}^{e}$ implies $\varphi \in D_{2}^{e}$.

Proof. Assume that $\varphi \in D_{2}^{e}$ and let $K, a,\left(a_{n}\right)_{n=1}^{\infty}$ and $\left(b_{n}\right)_{n=1}^{\infty}$ be as in the definition of the $D_{2}^{e}$-condition. Then

$$
\varphi_{n}(2 u) \leqslant K \varphi_{n}(u)+\varphi_{n}\left(2 a_{n}\right) \leqslant K \varphi_{n}(u)+K \varphi_{n}\left(a_{n}\right)
$$

for all $n \in \mathrm{N}$ and $u \in\left[0, b_{n}\right]$. Since $\left(\varphi_{n}\left(a_{n}\right)\right)_{n=1}^{\infty} \in e$ and $\varphi_{n}\left(b_{n}\right)\left\|e_{n}\right\|_{e}=a$ for all $n \in \mathrm{N}$, we conclude that $\varphi \in \delta_{2}^{e}$.

Assume now that $e \hookrightarrow c_{0}\left\{\left\|e_{n}\right\|_{e}\right\}$ and $\varphi \in \delta_{2}^{e}$. Let $K, a$ and $\left(c_{n}\right)_{n=1}^{\infty}$ be as in the definition of the $\delta_{2}^{e}$-condition. Define $b_{n} \geqslant 0$ such that $\varphi_{n}\left(b_{n}\right)\left\|e_{n}\right\|_{e}=a$ for any $n \in \mathrm{N}$. Without loss of generality we may assume that $c_{n} \leqslant \varphi_{n}\left(2 b_{n}\right)<\infty$ $(n \in \mathrm{N})$ because if $c_{n}>\varphi_{n}\left(2 b_{n}\right)$, then the inequality $\varphi_{n}(2 u) \leqslant \varphi_{n}\left(2 b_{n}\right)<c_{n}$ holds for any $u \in\left[0, b_{n}\right]$ automatically. So, for any $n \in \mathbf{N}$ there is $a_{n} \geqslant 0$ such that $\varphi_{n}\left(a_{n}\right)=c_{n}$. Since $\varphi_{n}\left(a_{n}\right)\left\|e_{n}\right\|_{e} \rightarrow 0$ as $n \rightarrow \infty$ and $\varphi_{n}\left(b_{n}\right)\left\|e_{n}\right\|_{e}=a$, 
we get $\varphi_{n}\left(a_{n}\right)<\varphi_{n}\left(b_{n}\right)$ for $n$ big enough (say for $n>k$ ), whence we conclude that $a_{n}<b_{n}$ for all $n>k$. Moreover, we have for any $n>k$ and $u \in\left[a_{n}, b_{n}\right]$,

$$
\begin{aligned}
\varphi_{n}(2 u) \leqslant K \varphi_{n}(u)+c_{n}=K \varphi_{n}(u) & +\varphi_{n}\left(a_{n}\right) \\
& \leqslant K \varphi_{n}(u)+\varphi_{n}(u)=(K+1) \varphi_{n}(u),
\end{aligned}
$$

which means that $\left(\varphi_{n}\right)_{n>k} \in D_{2}^{e}$. Since condition $\delta_{2}^{e}$ implies that

$$
\sup \left\{u \geqslant 0: \varphi_{n}(u)=0\right\}<\frac{1}{2} \sup \left\{u \geqslant 0: \varphi_{n}(u)<\infty\right\}
$$

for every $n \in \mathrm{N}$, we get $\varphi \in D_{2}^{e}$.

Remark 2. Any order continuous real Köthe sequence space $e$ is continuously embedded into $c_{0}\left\{\left\|e_{n}\right\|_{e}\right\}$.

Proof. It follows from $e \in(O C)$ that $\left\|\left(x_{k}\right)_{k \geqslant n}\right\|_{e} \rightarrow 0$ as $n \rightarrow \infty$ for any $x \in e$. In consequence, $\left|x_{n}\right|\left\|e_{n}\right\|_{e} \leqslant\left\|\left(x_{k}\right)_{k \geqslant n}\right\|_{e} \rightarrow 0$ as $n \rightarrow \infty$, that is, $x \in c_{0}\left\{\left\|e_{n}\right\|_{e}\right\}$. The continuity of the embedding follows from the last inequality.

LEMMA 4. If a real Köthe sequence space e is continuously embedded into both spaces $l^{\infty}$ and $c_{0}\left\{\left\|e_{n}\right\|_{e}\right\}, \varphi>0$ and all Orlicz functions $\varphi_{n}$ defining the Musielak-Orlicz function $\varphi=\left(\varphi_{n}\right)_{n=1}^{\infty}$ are the same, then $\varphi \in \delta_{2}^{e}$ is equivalent to $\varphi \in \Delta_{2}(0)$.

Proof. Let $\varphi_{n}=\varphi$ for any $n \in \mathbf{N}$. First, we will prove the implication $\delta_{2}^{e} \Rightarrow \Delta_{2}(0)$. By Lemma 3, we have $\varphi(2 u) \leqslant K \varphi(u)$ for $u \in\left[a_{n}, b_{n}\right], n \in \mathbf{N}$, where $\left(\varphi\left(a_{n}\right)\right)_{n=1}^{\infty} \in e, \varphi\left(b_{n}\right)\left\|e_{n}\right\|_{e}=a>0$ and $a_{n}<b_{n}$ for all $n \in \mathbf{N}$. Consequently, we have that $\varphi(2 u) \leqslant K \varphi(u)$ for any $u \in\left[\inf _{n} a_{n}\right.$, $\left.\sup _{n} b_{n}\right]$. The condition that $\left(\varphi\left(a_{n}\right)\right)_{n=1}^{\infty} \in e$ implies that $\varphi\left(a_{n}\right)\left\|e_{n}\right\|_{e} \rightarrow 0$ as $n \rightarrow \infty$. Since the assumption that $e \hookrightarrow l^{\infty}$ yields $\inf _{n}\left\|e_{n}\right\|_{e}>0$, we get $\varphi\left(a_{n}\right) \rightarrow 0$ as $n \rightarrow \infty$. Consequently, $\inf _{n} \varphi\left(a_{n}\right)=0$. By the assumption that $\varphi$ vanishes only at zero, we get $\inf _{n} a_{n}=0$. We can prove (although we need not to do this to have $\left.\varphi \in \Delta_{2}(0)\right)$ that $\sup _{n} b_{n}<\infty$. The assumption that $e \hookrightarrow l^{\infty}$ yields $\inf _{n}\left\|e_{n}\right\|_{e}>0$. Therefore, by $\varphi\left(b_{n}\right)\left\|e_{n}\right\|_{e}=a>0$, we have $\sup _{n} \varphi\left(b_{n}\right)<\infty$ and so $\sup _{n} b_{n}<\infty$. Consequently, $\varphi(2 u) \leqslant K \varphi(u)$ for all $u \in\left[0, \sup _{n} b_{n}\right]$ and $\operatorname{so} \varphi \in \Delta_{2}(0)$.

$\Delta_{2}(0) \Rightarrow \delta_{2}^{e}$. There are, by assumption, positive constants $K$ and $b$ such that $\varphi(2 u) \leqslant K \varphi(u)$ whenever $\varphi(u) \leqslant b$. By $e \hookrightarrow l^{\infty}$ there is $L>0$ such that $\left\|e_{n}\right\|_{e} \geqslant 1 / L$ for any $n \in \mathrm{N}$. Therefore, assuming that $\varphi(u)\left\|e_{n}\right\|_{e} \leqslant b / L$, we get $\varphi(u) \leqslant b /\left(L\left\|e_{n}\right\|_{e}\right) \leqslant b$. This yields the desired implication.

REMARK 3. There is a real Köthe sequence space $e$ such that $e \notin(O C)$ and $e \hookrightarrow c_{0}\left\{\left\|e_{n}\right\|_{e}\right\}$. 
Really, take an Orlicz function $\varphi$ such that $\varphi(1)=1, \varphi>0$ and $\varphi$ does not satisfy condition $\Delta_{2}(0)$. Then $\left\|e_{n}\right\|_{e}=1$ for any $n \in \mathrm{N}, L^{\varphi} \hookrightarrow c_{0}$ and $l^{\varphi} \notin(O C)$.

As an immediate consequence of Theorem 4 and Lemma 4, we get the following

COROLlary 4. Let a real Köthe sequence space $e \in(F P)$ and e be continuously embedded into both spaces $l^{\infty}$ and $c_{0}\left\{\left\|e_{n}\right\|_{e}\right\}$. Then the CalderónLozanovskĭ sequence space $e_{\varphi}^{\mathrm{C}}$ is C-rotund if and only if $\varphi \in \Delta_{2}(0)$, there is $u>0$ such that $\varphi(u) \inf _{n}\left\|e_{n}\right\|_{e}=1$ and $e$ is strictly monotone.

THeOREM 5. Let E be a real Köthe function space with the Fatou property and $\varphi$ be a Musielak-Orlicz function. The generalized complex CalderónLozanovskil space $E_{\varphi}^{\mathrm{C}}$ is uniformly $\mathrm{C}$-rotund if and only if $\varphi>0, \varphi \in \Delta_{2}^{E}$ and $E$ is uniformly monotone.

Proof. Every Köthe space $E$ which is uniformly monotone is order continuous (see [8], Proposition 2.1). The necessity of $\varphi \in \Delta_{2}^{E}$ follows from the fact that if $E \in(O C)$ and $\varphi \notin \Delta_{2}^{E}$, then $l^{\infty}$ embeds order isometrically into $E_{\varphi}$ (see [11]), and thus, $E_{\varphi}$ is not strictly monotone.

The necessity of $\varphi>0$ can be proved in the same way as in the case of an Orlicz function $\varphi$ in Theorem 2 of [4].

Now we can prove the necessity of the uniform monotonicity of $E$ for the uniform monotonicity of $E_{\varphi}$ (that is, for the uniform C-rotundity of $E_{\varphi}^{\mathrm{C}}$ ). Assume that $E$ is not uniformly monotone. Then there are two sequences $\left(x_{n}\right)$ and $\left(y_{n}\right)$ in $E^{+}$and $\varepsilon \in(0,1)$ such that $1 \geqslant\left\|y_{n}\right\|_{E} \geqslant \varepsilon,\left\|x_{n}\right\|_{E}=1, x_{n} \perp y_{n}$ (i.e. $\mu\left(\operatorname{supp} x_{n} \cap \operatorname{supp} y_{n}\right)=0$ ) for any $n \in \mathrm{N}$, and $\left\|x_{n}+y_{n}\right\|_{E} \rightarrow 1$ (see [16]). The condition $\varphi \in \Delta_{2}^{E}$ implies that $\varphi<\infty$, therefore we can find nonnegative functions $v_{n}, w_{n} \in L^{0}$ such that $x_{n}=\varphi \circ v_{n}$ and $y_{n}=\varphi \circ w_{n}$ for all $n \in \mathrm{N}$. Consequently, $\varrho_{\varphi}^{E}\left(v_{n}\right)=\left\|\varphi \circ v_{n}\right\|_{E}=\left\|x_{n}\right\|_{E}=1, \varrho_{\varphi}^{E}\left(w_{n}\right)=\left\|\varphi \circ w_{n}\right\|_{E}=$ $\left\|y_{n}\right\|_{E} \geqslant \varepsilon$ for all $n \in \mathrm{N}$ and,

$1 \leqslant \varrho_{\varphi}^{E}\left(v_{n}+w_{n}\right)=\left\|\varphi \circ\left(v_{n}+w_{n}\right)\right\|_{E}=\left\|\varphi \circ v_{n}+\varphi \circ w_{n}\right\|_{E}=\left\|x_{n}+y_{n}\right\|_{E} \rightarrow 1$

(the equality $\varphi \circ\left(v_{n}-w_{n}\right)=\varphi \circ v_{n}-\varphi \circ w_{n}$ follows by the fact that $v_{n} \perp w_{n}$ for all $n \in \mathrm{N}$, which is a consequence of the assumption that $x_{n} \perp y_{n}$ for any $n \in \mathrm{N})$. By the fact that if $\varrho_{\varphi}^{E}(z) \leqslant 1$, then $\varrho_{\varphi}^{E}(z) \leqslant\|z\|_{\varphi}^{E} \leqslant 1$ and if $\varrho_{\varphi}^{E}(z)>1$, then $\varrho_{\varphi}^{E}(z) \geqslant\|z\|_{\varphi}^{E} \geqslant 1$ for any $z \in E_{\varphi}$, we get $\left\|w_{n}\right\|_{\varphi}^{E} \geqslant \varepsilon$ for all $n \in \mathrm{N}$ and $\left\|v_{n}+w_{n}\right\|_{\varphi}^{E} \rightarrow 1$.

To finish the proof it is enough to apply Theorem 4.3 in [9] and Theorem 2.

COROLlARY 5. Let E be a real Köthe function space with the Fatou property and let $\varphi$ be an Orlicz function. The complex Calderón-Lozanovskiu space $E_{\varphi}^{\mathrm{C}}$ 
is uniformly C-rotund if and only if $\varphi>0, \varphi \in \Delta_{2}^{E}$ and $E$ is uniformly monotone.

Proof. Sufficiency. From our assumptions and Theorem 2 in [4] it follows that $E_{\varphi}$ is uniformly monotone and therefore $E_{\varphi}^{\mathrm{C}}$ is uniformly C-rotund.

Necessity. Any uniformly monotone Köthe space is order continuous, so we get the necessity of $\varphi \in \Delta_{2}^{E}$ from Corollary 3 . Consequently, we have also $\varphi<\infty$. If $E_{\varphi}^{\mathrm{C}}$ is uniformly C-rotund, then $E_{\varphi} \in(U M)$. This implies, since $\varphi<\infty$, that $E \in(U M)$ and $\varphi>0$ (Theorem 7 in [16] and Theorem 1 in [4]).

We say a Musielak-Orlicz function $\varphi$ satisfies condition $(*)_{e}$ if for each $\varepsilon \in(0,1)$ there exists $\eta \in(0,1)$ such that for all $n \in \mathbf{N}$ and $u \in \mathbf{R}_{+}$, satisfying $\left\|\varphi_{n}(u) e_{n}\right\|_{e} \leqslant 1-\varepsilon$, we have $\left\|\varphi_{n}((1+\eta) u) e_{n}\right\|_{e} \leqslant 1$ (see [12]).

Theorem 6. Let e be a real Köthe sequence space with the Fatou property. Then the generalized Calderón-Lozanovskiu sequence space $e_{\varphi}^{\mathrm{C}}$ is uniformly C-rotund if and only if $e \in(U M), \varphi \in \delta_{2}^{e}, \varphi>0$ and $\varphi$ satisfies condition $(*)_{e}$.

Proof. It is enough to apply Theorem 5.3 from [12] and our Theorem 2.

Although the class of Orlicz-Lorentz spaces is a subclass of CalderónLozanovskii spaces (we get it taking for $E$ (resp. $e$ ) the Lorentz space $\Lambda_{\omega}$ (resp. $\left.\lambda_{\omega}\right)$ ) in this special interesting case, criteria for the monotonicity and Crotundity properties are clearer and more specified. Therefore, it is of interest to present them, especially for the reason that we need to complete some results from [14] on the monotonicity properties for Lorentz spaces $\Lambda_{\omega}$ and $\lambda_{\omega}$. For the definition of these spaces see [18], [15], [3] and [14].

THEOREM 7. Let $(T, \Sigma, \mu)$ be a non-atomic, $\sigma$-finite and complete measure space. The Orlicz-Lorentz function space $\Lambda_{\varphi, \omega}^{\mathrm{C}}$ is $\mathrm{C}$-rotund if and only if the weight function $\omega$ is strictly positive, $\int_{0}^{\gamma} \omega(t) d t=\infty$ if $\gamma=\infty, \varphi>0$, and $\varphi \in \Delta_{2}\left(\mathbf{R}_{+}\right)$if $\mu(T)=\infty$ (resp. $\varphi \in \Delta_{2}(\infty)$ if $\left.\mu(T)<\infty\right)$.

Proof. We need only prove that our assumptions are necessary and sufficient for strict monotonicity of the real space $\Lambda_{\varphi, \omega}$. This was partially proved in [14].

Necessity. If $\varphi \notin \Delta_{2}\left(\mathbf{R}_{+}\right)$when $\mu(T)=\infty$ (or $\varphi \notin \Delta_{2}(\infty)$ if $\mu(T)<\infty$ ), then $\Lambda_{\varphi, \omega}$ contains an order isometric copy of $l^{\infty}$ (see [15]), so $\Lambda_{\varphi, \omega}$ is not strictly monotone. If $\gamma=\infty$ and $\int_{0}^{\gamma} \omega(t) d t<\infty$, then $\Lambda_{\varphi, \omega}$ also contains an order isometric copy of $l^{\infty}$ (see [15]), whence $\Lambda_{\varphi, \omega}$ is not strictly monotone. Assume that the condition that $\varphi>0$ is not satisfied, that is, $a_{\varphi}:=\sup \{u \geqslant$ $0: \varphi(u)=0\}>0$. Look at the function $x=a \chi_{A}$ with $a>a_{\varphi}, A \in \Sigma$, $0<\mu(A)<\infty, \mu(T \backslash A)>0$ and $\|x\|_{\varphi, \omega}=1$. Define $y=x+a_{\varphi} \chi_{T \backslash A}$. 
Then $x^{*}=a \chi_{[0, \mu(A)]}$ and $y^{*}=a \chi_{[0, \mu(A)]}+a_{\varphi} \chi_{(\mu(A), \infty)}$. Since

$$
\begin{aligned}
\varrho_{\varphi, \omega}(y) & =\int_{0}^{\infty} \varphi\left(y^{*}(t)\right) \omega(t) d \mu=\int_{0}^{\mu(A)} \varphi(a) \omega(t) d \mu \\
& =\int_{0}^{\infty} \varphi\left(x^{*}(t)\right) \omega(t) d \mu=\varrho_{\varphi, \omega}(x) \leqslant 1,
\end{aligned}
$$

we get $\|y\|_{\varphi, \omega} \leqslant 1$. Now, the inequality $0 \leqslant x \leqslant y$ and the equality $\|x\|_{\varphi, \omega}=$ 1 , yields $\|y\|_{\varphi, \omega} \geqslant 1$. Consequently $\|y\|_{\varphi, \omega}=1$, which means that $\Lambda_{\varphi, \omega}$ is not strictly monotone.

Assume now that $\omega$ is not strictly positive. Then $b(\omega):=\sup \{t>0$ : $\omega(t)>0\}<\gamma$, where $\gamma=\mu(T)$. Let $A \in \Sigma$ and $a>0$ be such that $b(\omega)<$ $\mu(A)<\gamma$ and such that the function $x=a \chi_{A}$ satisfies $\|x\|_{\varphi, \omega}=1$. Choose $0<c<a$ and define $y=x+c \chi_{T \backslash A}$. Then $y^{*}=a \chi_{[0, \mu(A)]}+c \chi_{(\mu(A), \gamma)}$. Consequently, since $\omega(t)=0$ for $t \in(\mu(A), \gamma)$, we get $\varrho_{\varphi, \omega}(y)=\varrho_{\varphi, \omega}(x)$, whence we easily deduce that $\|x\|_{\varphi, \omega}=\|y\|_{\varphi, \omega}=1$, which means that $\Lambda_{\varphi, \omega}$ is not strictly monotone.

Sufficiency. Assume that the assumptions on $\varphi$ and $\omega$ are satisfied, $0 \leqslant y \leqslant$ $x, x \neq y$, and $\|x\|_{\varphi, \omega}=1$. Then $\varrho_{\varphi, \omega}(x)=1$. Moreover, $y^{*} \leqslant x^{*}$ and the condition $y^{*} \neq x^{*}$ follows by the assumption that $\int_{0}^{\gamma} \omega(t) d t=\infty$ (if $\gamma=\infty$ ) because the last condition yields that $d_{x}(\lambda)=\mu(\{t: x(t)>\lambda\})<\infty$ for any $\lambda>0$ (see [18]). Consequently, by the fact that $\varphi>0$ and by strict positivity of $\omega$, we get $\varrho_{\varphi, \omega}(y)<\varrho_{\varphi, \omega}(x)=1$. Since $\varphi \in \Delta_{2}\left(R_{+}\right)$if $\mu(T)=\infty$ (or $\varphi \in \Delta_{2}(\infty)$ if $\left.\mu(T)<\infty\right)$, we get $\|y\|_{\varphi, \omega}<1$, which means that $\Lambda_{\varphi, \omega}$ is strictly monotone.

THEOREM 8. The Orlicz-Lorentz sequence space $\lambda_{\varphi, \omega}^{\mathrm{C}}$ is $\mathrm{C}$-rotund if and only if $\varphi$ satisfies the $\Delta_{2}(0)$-condition, there is $u_{0}>0$ such that $\varphi\left(u_{0}\right) \omega_{1}=1$ and $\sum_{n=1}^{\infty} \omega_{n}=\infty$.

Proof. Necessity. Assume that $\sum_{n=1}^{\infty} \omega_{n}<\infty$. We will show that $\lambda_{\varphi, \omega}$ contains then an order isometric copy of $l_{\infty}$. Divide $\mathrm{N}$ into a sequence $\left(N_{k}\right)$ of infinite and pairwise disjoint sets and define $x=a \chi_{\mathrm{N}}, x_{k}=a \chi_{N_{k}}(k \in \mathrm{N})$, where $a>0$ is chosen in such a way that $\sum_{n=1}^{\infty} \varphi(a) \omega_{n}=1$. It is obvious that $x^{*}=x_{k}^{*}=a \chi_{\mathrm{N}}$ for all $k \in \mathrm{N}$. Therefore, $\varrho_{\varphi, \omega}(x)=\varrho_{\varphi, \omega}\left(x_{k}\right)=1$, whence $\|x\|_{\varphi, \omega}=\left\|x_{k}\right\|_{\varphi, \omega}=1$ for all $k \in \mathrm{N}$. Since $x=\sum_{k=1}^{\infty} x_{k}$ pointwise, the operator $P: l_{\infty} \rightarrow \lambda_{\varphi, \omega}$ defined by $c=\left(c_{k}\right) \longmapsto P c=\sum_{k=1}^{\infty} c_{k} x_{k}$ defines a linear order isometry, which follows from the fact that

$$
\varrho_{\varphi, \omega}\left(P c /\|c\|_{\infty}\right) \leqslant 1 \quad \text { and } \varrho_{\varphi, \omega}\left(P c /\left(\lambda\|c\|_{\infty}\right)\right)>1
$$

for any $\lambda \in(0,1)$. Thus $\lambda_{\varphi, \omega}$ is not strictly monotone, whence $\lambda_{\varphi, \omega}^{C}$ is not C-rotund. 
If we prove the necessity of the fact that $\varphi\left(u_{0}\right) \omega_{1}=1$ for some $u_{0}>0$, the remaining part of the proof of the necessity can be done as in the proof of Theorem 7, because for every $x \in B\left(\lambda_{\varphi, \omega}\right)$, we have $|x(i)| \in\left[0, u_{0}\right]$ for any $i \in \mathrm{N}$ and the behavior of $\varphi$ outside of the interval $\left[0, u_{0}\right]$ has no influence on strict monotonicity of $\lambda_{\varphi, \omega}$. In the proof of Theorem 7, we referred to [15] in order to prove the necessity of $\varphi \in \Delta_{2}\left(R_{+}\right)$(or $\varphi \in \Delta_{2}(\infty)$ ) for strict monotonicity of $\Lambda_{\varphi, \omega}$. In the sequence case we should refer to [3], where it has been proved that $\lambda_{\varphi, \omega}$ contains an order isometric copy of $l_{\infty}$ whenever $\varphi \notin \Delta_{2}(0)$.

Assume that there is no $u_{0}>0$ such that $\varphi\left(u_{0}\right) \omega_{1}=1$. Consequently, for $\alpha:=\sup \{u>0: \varphi(u)<\infty\}$, we have $\varphi(\alpha) \omega_{1}<1$. Defining $x=\alpha e_{1}$ and $y=\alpha e_{1}+c e_{2}$, where $0<c<\alpha$ satisfies the inequality $\varphi(\alpha) \omega_{1}+\varphi(c) \omega_{2} \leqslant 1$, we have $x^{*}=x, y^{*}=y$ and $\max \left(\varrho_{\varphi, \omega}(x), \varrho_{\varphi, \omega}(y)\right) \leqslant 1$. Moreover, we have $\varrho_{\varphi, \omega}(\beta x)=\varrho_{\varphi, \omega}(\beta y)=\infty$ for any $\beta>1$. Consequently, $\|x\|_{\varphi, \omega}=\|y\|_{\varphi, \omega}=$ 1 . Since $0 \leqslant x \leqslant y$ and $x \neq y$, this means that $\lambda_{\varphi, \omega}$ is not strictly monotone.

Sufficiency. Assuming that $0 \leqslant y \leqslant x, x \in S\left(\lambda_{\varphi, \omega}\right)$ and $y \neq x$, the assumptions yield that $0 \leqslant y^{*} \leqslant x^{*}$ and $y^{*} \neq x^{*}$. Since the weighted $l_{1}$-space $l_{1}\left(\left\{\omega_{n}\right\}\right)$ is strictly monotone, we get $\varrho_{\varphi, \omega}(y)<\varrho_{\varphi, \omega}(x)=1$. The assumption that $\varphi \in \Delta_{2}(0)$ yields $\|y\|_{\varphi, \omega}<1$, that is, $\lambda_{\varphi, \omega}$ is strictly monotone.

We say that the weight function $\omega$ is regular if there exist $K>1$ such that $S(2 t) \geqslant K S(t)$ for all $t \in(0, \gamma / 2)$, where $S(t)=\int_{0}^{t} \omega(s) d s$.

THEOREM 9. The Orlicz-Lorentz function space $\Lambda_{\varphi, \omega}^{\mathrm{C}}$ is uniformly $\mathrm{C}$-rotund if and only if $\varphi>0, \varphi$ satisfies the $\Delta_{2}\left(\mathbf{R}_{+}\right)$-condition if $\mu(T)=\infty$ (resp. the $\Delta_{2}(\infty)$-condition if $\left.\mu(T)<\infty\right)$ and $\omega$ is regular.

PROOF. This is a consequence of Theorem 2 and the results from [14], which establish that $\Lambda_{\varphi, \omega}$ is uniformly monotone if and only if our assumptions are satisfied (see [14]).

THEOREM 10. Let $\varphi$ be an Orlicz function. The Orlicz-Lorentz sequence space $\lambda_{\varphi, \omega}^{C}$ is uniformly $\mathrm{C}$-rotund if and only if there is $u_{0}>0$ such that $\varphi\left(u_{0}\right) \omega_{1}=1, \varphi$ satisfies the $\Delta_{2}(0)$-condition and the weight sequence $\omega$ is regular.

Proof. On the basis of the results from [12] and Theorem 2, the argumentation from the previous proof can be repeated here.

ACKNOWLEDGMENT. The authors thank the Referee for valuable remarks and suggestions. 


\section{REFERENCES}

1. Akcoglu, M. A., Sucheston, L., On uniform monotonicity of norms and ergodic theorems in function spaces, Rend. Circ. Mat. Palermo (2) Suppl. 8 (1985), 325-335.

2. Birkhoff, G., Lattice Theory, Amer. Math. Soc., Providence, R. I., 1967.

3. Cerdá, J., Hudzik, H., Kamińska, A., Mastyło, M., Geometric properties of symmetric spaces with applications to Orlicz-Lorentz spaces, Positivity 2 (1998), 311-337.

4. Cerdá, J., Hudzik, H., Mastyło, M., On the geometry of some Calderón-Lozanovskil spaces, Indag. Math. (N.S.) 6(1) (1995), 35-49.

5. Chen, S., Geometry of Orlicz Spaces, Dissertationes Math. 356 (1996), 1-204.

6. Chen, S., Płuciennik, R., A note on H-points in Köthe-Bochner spaces, Acta Math. Hungar. 94(1-2) (2002), 59-66.

7. Dilworth, S. J., Complex convexity and the geometry of Banach spaces, Math. Proc. Cambridge Philos. Soc. 99 (1986), 495-506.

8. Dominguez, T., Hudzik, H., Lopez, G., Mastyło, M., Sims, B., Complete characterizations of the Kadec-Klee properties in Orlicz spaces, Houston J. Math. 29(4) (2003), 1027-1044.

9. Foralewski, P., On topological and geometrical structure of generalized Calderón-Lozanovski spaces, Ph.D. Dissertation, Adam Mickiewicz University, Poznań, 1997.

10. Foralewski, P., On some geometric properties of generalized Calderón-Lozanovskiŭ spaces, Acta Math. Hungar. 80(1-2) (1998), 55-66.

11. Foralewski, P., Hudzik, H., Some basic properties of generalized Calderón-Lozanovskiŭ spaces, Collect. Math. 48(4-6) (1997), 523-538.

12. Foralewski, P., Hudzik, H., On some geometrical and topological properties of generalized Calderón-Lozanovskil sequence spaces, Houston J. Math. 25(3) (1999), 523-542.

13. Globevnik, J., On complex strict and uniform convexity, Proc. Amer. Math. Soc. 47 (1975), $175-178$.

14. Hudzik, H., Kamińska, A., Monotonicity properties of Lorentz spaces, Proc. Amer. Math. Soc. 123(9) (1995), 2715-2721.

15. Hudzik, H., Kamińska, A., Mastyło, M., Geometric properties of some Calderón-Lozanovski spaces and Orlicz-Lorentz spaces, Houston J. Math. 23(3) (1996), 639-663.

16. Hudzik, H., Kamińska, A., Mastyło, M., Monotonicity and rotundity properties in Banach lattices, Rocky Mountain J. Math. 30(3) (2000), 933-950.

17. Hudzik, H., Kurc, W., Monotonicity properties of Musielak-Orlicz spaces and dominated best approximation in Banach lattices, J. Approx. Theory 95 (1998), 353-368.

18. Kamińska, A., Some remarks on Orlicz-Lorentz spaces, Math. Nachr. 147 (1990), 29-38.

19. Kurc, W., Strictly and uniformly monotone Musielak-Orlicz spaces and applications to best approximation, J. Approx. Theory 69(2) (1992), 173-187.

20. Kurc, W., Strictly and uniformly monotone sequential Musielak-Orlicz spaces, Collect. Math. 50(1) (1999), 1-17.

21. Liu, L., On some local and global geometric properties of Musielak-Orlicz spaces, Ph.D. Dissertation, Adam Mickiewicz University, Poznań, 2001.

22. Maligranda, L., Orlicz spaces and interpolation, Seminars in Math. 5, Campinas, 1989.

23. Thorp, E., Whitley, R., The strong maximum modulus theorem for analytic functions into a Banach space, Proc. Amer. Math. Soc. 18 (1967), 640-646.

24. Wu, C., Sun, H., On the complex convexity of Musielak-Orlicz spaces, Comment. Math. Univ. Carolin. 30 (1989), 397-408.

25. Wu, C., Sun, H., On the complex extreme points and complex strictly rotundity of MusielakOrlicz spaces, J. Systems Sci. Math. 7 (1987), 7-13.

26. Wu, C., Sun, H., On the complex uniformly rotundity of Musielak-Orlicz spaces, J. Nordheast Math. 4 (1988), 389-396. 
27. Wang, T., Teng, Y., Complex locally uniform rotundity of Musielak-Orlicz spaces, Science of China 30(1) (2000), 24-31.

FACULTY OF MATHEMATICS AND COMPUTER SCIENCE ADAM MICKIEWICZ UNIVERSITY

UMULTOWSKA 87

61-614 POZNAŃ

POLAND

E-mail: hudzik@amu.edu.pl
INSTITUTE OF MATHEMATICS

SZCZECIN UNIVERSITY

WIELKOPOLSKA 15

70-451 SZCZECIN

POLAND

E-mail: narloch@sus.univ.szczecin.pl 